\title{
A Case of Maxillary Sinus Cholesteatoma Originating from the Retromaxillary Sinus Wall
}

\author{
Jun Myung Lee, Nam Gyu Ryu, Ick Soo Choi \\ Department of Otorhinolaryngology-Head and Neck Surgery, Inje University College of Medicine, \\ Ilsanpaik Hospital, Goyang, Korea \\ Email: leochoics@gmail.com
}

Received 23 June 2015; accepted 14 August 2015; published 17 August 2015

Copyright (C) 2015 by authors and Scientific Research Publishing Inc.

This work is licensed under the Creative Commons Attribution International License (CC BY). http://creativecommons.org/licenses/by/4.0/

(c) (i) Open Access

\begin{abstract}
Cholesteatomas are often observed in the middle ear or mastoid cavity. However, cholesteatomas in areas other than the middle ear or mastoid are extremely rare and thus are not often reported in the literature. We recently treated an 18-year-old female patient with an incidentally detected maxillary sinus mass. A paranasal sinus computed tomography examination revealed a cystic lesion in the left maxillary sinus infiltrating the posterolateral walls of the left maxillary sinus. An endoscopic operation was performed for definitive diagnosis. Histopathological examination of the mass confirmed the diagnosis of cholesteatoma. Despite the rarity of paranasal cholesteatoma in comparison with cholesteatomas in the tympanum, a few cases involving the frontal sinus have been reported. However, retromaxillary cholesteatoma is even less common than sinus cholesteatoma and is reported rarely. To our knowledge, this is the first reported case of a maxillary cholesteatoma originating from the retromaxillary area. For this reason, we present this case along with a literature review.
\end{abstract}

\section{Keywords}

Cholesteatoma, Paranasal Sinus, Retromaxillary Sinus, Paranasal Sinus Computed Tomography

\section{Introduction}

A cholesteatoma is a mass formed by keratin layers within a hyper-keratinized squamous epithelium that partially or entirely replaces the normal mucous membrane [1]. These masses are commonly formed in the middle ear or mastoid cavity. However, there are reports of cholesteatomas originating outside the middle ear or masto- 
id cavity. Cholesteatomas in the maxillary sinus are rare and those in the retromaxillary sinus are extremely rare [2]. A few hypotheses have tried to explain the likely causes of nasal sinus cholesteatomas, but a clear cause has not been identified yet [2] [3].

Symptoms of nasal sinus cholesteatoma depend on the location and can include rhinorrhea, nasal obstruction, facial edema, tenderness on palpation, exophthalmos, and oculomotor dysfunction [2] [4]. This case report describes the histopathological characteristics of a cholesteatoma in the retromaxillary space that is incidentally detected by brain computed tomography (CT). A mass in the retromaxillary space, which grew while pushing the maxillary sinus outward, was removed. We report the findings in this case and provide a literature review.

\section{Case}

An 18-year-old woman presented to the hospital with persistent dizziness and underwent a brain CT examination. The CT images incidentally showed a mass in the left maxillary sinus, which appeared as a radiopaque lesion containing relatively homogeneous soft tissues inside the left maxillary sinus. The patient was referred to the department of otorhinolaryngology for further evaluation, and her dizziness was found to be secondary to orthostatic hypotension. She denied experiencing headache, facial pain, nasal obstruction, and toothache. She also reported no other medical history or familial disorders.

Endoscopic examination revealed hypertrophy of both inferior turbinates, deviation of the nasal septum, and absence of nasal polyps. The blood test did not show any abnormal findings. For detailed characterization of nasal sinus mass, a paranasal sinus (PNS) CT examination was performed. The PNS CT images showed a cystic lesion in the left maxillary sinus (MS) infiltrating into the posterolateral walls of the left MS. The mass showed contrast enhancement in the outer edges, with radiopaque soft tissue shading partially inside. There was no maxillary sinus expansion except for a bony defect on the posterolateral wall of the left MS (Figure 1).

To make a definitive diagnosis, an endoscopic operation was performed. After widening the natural ostium, we explored the lesion endoscopically. In the left MS, a fibrous, homogeneous membrane was observed, appearing thin and solid like an eggshell. The posterolateral bony wall of the left MS was protruding into the sinus, forming a part of the cystic wall. The cystic mass pushed into the sinus from its posterior portion and contained a cavity filled with a keratin-like material. The mass was removed and the findings of the histologic review were consistent with cholesteatoma (Figure 2).

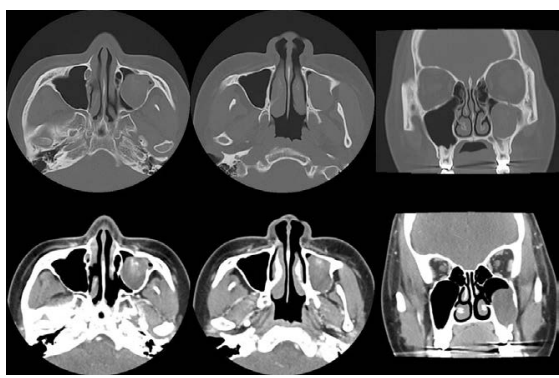

Figure 1. Cystic lesion invading posterior and lateral left maxillary wall.

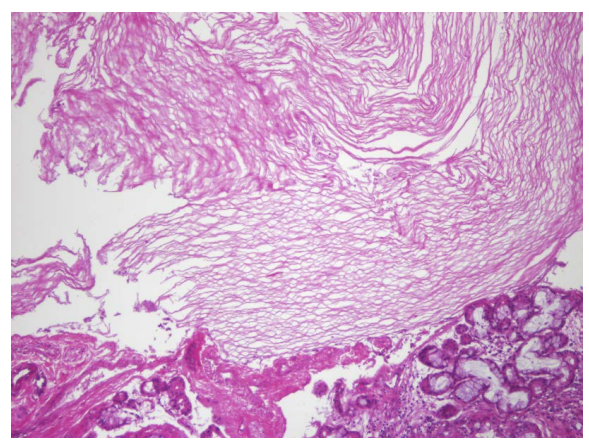

Figure 2. The specimen shows aggregation of keratin debris and normal seromucinous glands $(\mathrm{HE}, \times 100)$. 


\section{Discussion}

Cholesteatomas can develop in the skin, breast, kidney, central nervous system, and inside the cranium [4]-[6]. Cholesteatomas of the head and neck mostly involve the ears. Dead cells abnormally accumulate behind the damaged tympanic membrane from repeated infections or injuries and form a pearl-colored mass. Cholesteatomas within the paranasal sinus are extremely rare compared to those in the temporal bone [4] [5]. Cholesteatomas occur most frequently in the frontal nasal sinus, which was still lower frequency. Thus, cholesteatomas in MS are even less common and have been rarely reported. One case of maxillary sinus cholesteatoma was reported in 1999, in which the cholesteatoma was accompanied by tooth extraction in the maxillary sinus [7]. The inner layer of the paranasal sinuses consists of respiratory epithelium with pseudostratified ciliated columnar epithelium. If this respiratory epithelium is replaced partially or entirely with keratinized squamous epithelium, it would form a cholesteatoma [1].

There are several hypotheses explaining the development of cholesteatomas inside the paranasal sinuses. The theory of "congenital epithelial rest" is widely recognized and suggests that cholesteatomas can originate from the remaining epithelial tissues after the embryonic developmental process. If the division of the two ectodermal surfaces near the 5th week of embryo development is incomplete, the neural groove closure will not occur normally. Therefore, some portion of the epithelial tissues remains in between these two surfaces, leading to cholesteatoma development [2] [3] [8] [9]. Further theories include "the implantation theory", contending that epithelial tissues are directly injected due to injuries such as surgery [4] [9]; the "immigration theory" that hyperkeratinized squamous epithelium in a normal position moves inside the sinus through the sinus opening [1] [9]; and the "metaplasia theory" that chronic infection causes metaplasia of the normal membrane in the sinus [1] [9]-[11]. Although none of these theories clearly explain the development of cholesteatomas, they provide insight into the general understanding of cholesteatoma formation in the nasal sinus.

In this case, the patient had no history of surgery or previous sinus inflammation; therefore, the cholesteatoma appeared to originate from the remnant epithelial tissue and grew from the lateral wall of the retromaxillary sinus [2] [3] [8]. As intranasal sinus cholesteatomas are rare, it is difficult to diagnose them without histopathological examination of samples obtained through surgery. However, medical history, physical examination findings, and imaging studies can provide reasonable suspicion [2].

Symptoms develop as the cholesteatoma expands into the nasal sinus, and the symptoms themselves may vary according to the location of the cholesteatoma. If the cholesteatoma expands towards the ostiomeatal complex, rhinorrhea and sinusitis may occur. If it infiltrates into the nasal vestibule, nasal obstruction may be possible. When the cholesteatoma invades the anterolateral wall of the antrum, facial swelling and tenderness on palpation could appear, while invasion into the inferior part of the antrum may lead to palate swelling. If it extends into the upper part of maxillary sinus, exophthalmos, conjunctival swelling, and oculomotor dysfunction could develop [2] [4].

In CT images, the characteristic intra-sinus cholesteatoma shows a relatively homogeneous, expansile lesion with osteoclasia in the surrounding area. If the image shows an enhancing cystic mass involving the paranasal sinuses and infiltrating the paranasal bone, it may indicate a cholesteatoma of the paranasal sinus. Although this feature has limited diagnostic value because intra-maxillary sinus mucocele or fungal sinusitis present with similar findings, such an examination is relatively convenient and provides an easy approach to detect the lesion characteristics [9] [12]. Thus, it could be an appropriate procedure for preliminary diagnosis and follow-up examinations [7].

Magnetic resonance imaging (MRI) is helpful in the diagnosis and decision making due to the excellent soft tissue projection. However, MRI examinations are costly and therefore only performed if paranasal sinus cholesteatoma is suspected [7]. Treatment of cholesteatoma involves complete removal of the cholesteatoma and the wrapping sac [6]. There are no established treatment guidelines for intranasal cholesteatoma. Several researchers recommended the Caldwell-Luc operation for complete removal of intra-sinus cholesteatoma [1] [9] [13]. The Caldwell-Luc operation would have been a consideration in this case if the cholesteatoma was larger and had further expanded into the medial side. Fortunately, the cholesteatoma in this case was not huge and did not extend inside the sinus and showed minimal bony erosion. This made lesion removal by an endoscopic sinus operation possible. However, to ensure complete removal and easier follow-up in the outpatient clinic, a large antrostomy was performed. Postoperative relapse has not been reported yet. In this case, the cholesteatoma seemed to originate from the retromaxillary space and was diagnosed as cholesteatoma. Further follow-up observations are necessary to determine the natural course of this retromaxillary cholesteatoma. 


\section{Conclusion}

Retromaxillary sinus cholesteatoma is extremely rare and may have no specific symptoms until it grows near the surrounding structures. It may show an enhancing cystic mass involving the paranasal sinuses and infiltrating the paranasal bone in PNS CT. Paranasal cholesteatoma should be removed completely by surgery because of its expansile characteristics.

\section{Conflicts}

The authors have no conflicts of interest to declare in relation to this article.

\section{References}

[1] Pogorel, B.S. and Budd, E.G. (1965) Cholesteatoma of the Maxillary Sinus. Archives of Otolaryngology, 82, 532-534. http://dx.doi.org/10.1001/archotol.1965.00760010534016

[2] Hartman, J.M., Stankiewicz, J.A. and Maywood, I.L. (1991) Cholesteatoma of the Paranasal Sinuses: Case Report and Review of the Literature. Ear, Nose \& Throat Journal, 70, 719-725.

[3] Sadoff, R.S. and Pliskin, A. (1989) Cholesteatoma (Keratoma) of the Maxillary Sinus: Report of a Case. Journal of Oral and Maxillofacial Surgery, 47, 873-876. http://dx.doi.org/10.1016/S0278-2391(89)80052-7

[4] Hopp, M.L. and Montgomery, W.W. (1984) Primary and Secondary Keratomas of the Frontal Sinus. Laryngoscope, 94, 628-632. http://dx.doi.org/10.1288/00005537-198405000-00010

[5] Paaske, P.B. (1984) Cholesteatoma of the Maxillary Sinus. Journal of Laryngology and Otology, 98, 539-541. http://dx.doi.org/10.1017/S0022215100147048

[6] Puttamadaiah, G.M., Vijayashree, M.S., Viswanatha, B. and Kaur, J. (2014) Cholesteatoma of Maxillary Sinus Mimicking Malignancy. Research in Otolaryngology, 3, 57-59.

[7] Sang, H.P., Seung, H.B., Tae, H.S. and Young, J.C. (1999) Cholesteatoma of the Maxillary Sinus. Korean Journal of Otolaryngology-Head and Neck Surgery, 42, 522-525.

[8] Baxter, J.J.R. (1966) Cholesteatoma of the Maxillary Antrum. Journal of Laryngology and Otology, 80, $1059-1061$. http://dx.doi.org/10.1017/S002221510006638X

[9] Viswanatha, B., Nayak, L.K. and Karthik, S. (2007) Cholesteatoma of the Maxillary Sinus. Ear, Nose \& Throat Journal, 86, 351-353.

[10] Mills, P.C. and Sycamore, E.M.K. (1958) Cholesteatoma of the Maxillary Antrum. Journal of Laryngology and Otology, 72, 580-583. http://dx.doi.org/10.1017/S0022215100054347

[11] Das, S.K. (1971) Cholesteatoma of the Maxillary Antrum. Journal of Laryngology and Otology, 85, 397-400. http://dx.doi.org/10.1017/S0022215100073588

[12] Valvassori, G.E. (2003) Imaging of the Temporal Bone. In: Glasscock, M.E. and Gulya, A.J., Eds., Shambaugh’s Surgery of the Ear, 5th Edition, B C Decker, Hamilton, 227-259.

[13] Yağci, A.B., Kara, C.O., Karabulut, N., et al. (2003) Horseshoe Maxillary Sinus: CT of a Unique Case with Cholesteatoma. European Journal of Radiology Extra, 48, 5-7. http://dx.doi.org/10.1016/S1571-4675(03)00078-6 